\title{
Malignant Cardiac Paraganglioma
}

National Cancer Institute

\section{Source}

National Cancer Institute. Malignant Cardiac Paraganglioma. NCI Thesaurus. Code C5392.

A paragang lioma of the heart that metastasizes to regional or distant anatomic sites. 